\title{
Steady Solution for Thermal Convection within the Earth's Mantle
}

\author{
By \\ Jun IRIYama, Kazuo Fukuta \\ Department of Fundamental Science, Faculty of Engineering, \\ Chubu Institute of Technology, Kasugai, Aichi, Japan \\ and \\ Yasuo SHIMAzU \\ Department of Earth Sciences, Faculty of Science, \\ Nagoya University, Nagoya, Japan
}

\begin{abstract}
A linear stability problem for the mantle of the earth is considered. Upper and lower mantle convection currents are assumed to reflect a rapid change in the viscosity at transition zone. The theory of mantle convection proposed by TOZER is adopted as a basis for the present investigation. For appropriate values of physical parameters it is found that the mantle should be in a convective state. Using the minimum temperature assumption, the internal temperature distribution in the presence of mantle convection current is considered.

The temperature at the bottom of the upper mantle convection cell is about $1450-1650^{\circ} \mathrm{K}$ regardless of conduction transfer and the thickness of the convecting zone. The temperature gradient within the upper mantle convecting zone differs very little from the adiabatic gradient. The temperature at the core-mantle boundary is about $2100-3800^{\circ} \mathrm{K}$.
\end{abstract}

\section{$\S 1$. Theoretical Background}

The onset of convection at some zone within a chemically homogeneous planet is governed by the steepness of the temperature gradient $d T / d r$ relative to the adiabatic gradient $(d T / d r)_{a d}$ at that zone. If $d T / d r$ were less than or equal to $(d T / d r)_{a d}$ under these conditions, then there would be no energy transfer by convection. Convection current carriers large amounts of energy from the interior of the earth, and therefore a steady situation will be realized eventually, in which both conduction and convection compete as heat transport mechanism (CoX and GruLI, 1968). The actual $T(r)$ curve within the convective zone has a slope intermediate between that of the conductive $T(r)$ curve and that of the adiabatic $T(r)$ curve.

We assume that the thermal convection will occur if the Rayleigh number (the dimensionless parameter)

$$
R a=g \alpha d^{3} \delta T / \kappa \nu
$$

SMLES Contribution No. 35. exceeds a critical value of 2600 , where $g$ is the acceleration of gravity, $\alpha$ the thermal coefficient of expansion $\left(=2 \times 10^{-5} / \mathrm{deg}\right), d$ the thickness of the layer, $\delta T$ the temperature difference between the upper and lower boundary of the considering layer calculated from conduction theory, $\kappa$ the thermal diffusivity, and $\nu$ the kinematic viscosity.

The theory of mantle convection proposed by Tozer (1967) is adopted here as a basis for the present calculation. We assume that convection is to reduce the temperature at the bottom of the convecting layer a minimum. If heat is fully transported to the upper part of the layer by convection current, then the temperature at the lower boundary of the convecting layer will become a minimum. The ratio of the heat brought to the upper surface of a convecting system to the heat that is brought by conduction is given in terms of the Nusselt number, a dimensionless quantity defined as (e.g., KNOPOFF, 1964) 


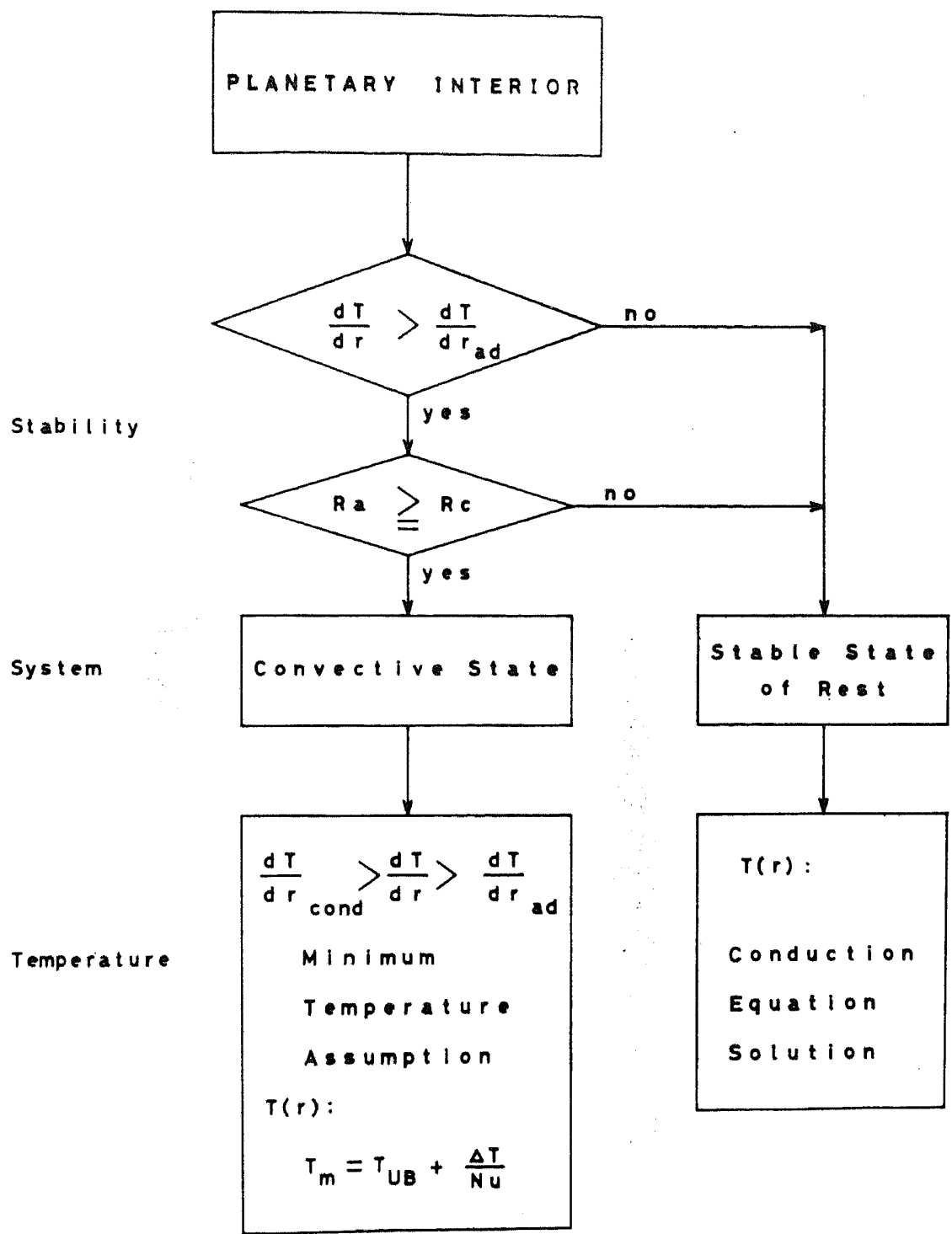

Fig. 1. An outline of the calculation.

$$
N u=H /\left(\kappa \rho C_{p} \delta T / d\right)
$$

where $H$ is the total heat escaping at the upper surface and $\rho$ the density. Thus, the temperature at the bottom of convection cell will decrease in proportion to the Nusselt number in the system and is (TOZER, 1967)

$$
T_{m}=T_{U B}+\delta T / N u
$$

where $T_{U B}$ is the temperature at the upper boundary of the fluid layer calculated from conduction theory. The Nusselt number is calculated from the Rayleigh number in the convecting system by using the empirical results by SILvESTON (1958).

An outline of the present calculation is shown in Figure 1.

\section{§ 2. Model}

We consider the mantle convection model under the fixed boundary conditions and internal heat generation. The upper and lower mantle convection currents are assumed 
to reflect a rapid change in the viscosity at transition zone, i.e., Bullen's region $\mathrm{C}$. Three models are considered with respect to the conductive temperature distribution within the mantle. These are classified into the following types: (1) high temperature types (models H1 and H2; IrIYAMA, 1970, his models RC-U2 and RH-U2), (2) low temperature types (models L1 and L2), and (3) oceanic and shield types (models OCEAN and SHIELD; CLARK and RINGWOOD, 1964). These are illustrated in Figures 2, 3, 4, 5, and 6.

The thermal conductivity of the medium is given by

$$
K_{T}=C+16 n^{2} S T^{3} / 3 \varepsilon
$$

where $C$ is the ordinary lattice conductivity $\left(=2.5 \times 10^{5} \mathrm{ergs} / \mathrm{cm} / \mathrm{sec} / \mathrm{deg}\right), n$ the refractive index $(=1.7), S$ the Stefan-Boltzmann constant, and $\varepsilon$ the opacity $(10 / \mathrm{cm})$.

We assume that the shear viscosity of the medium is (TOZER, 1967)

$$
\eta=700 T \exp (E / k T)
$$

where $E$ is the activation energy, and $k$ the Boltzmann's constant. It is supposed that a rapid change of the viscosity occurs in the transition zone of the mantle. Thus, in the calculations the activation energy within upper mantle convection zone is set equal to $4.0 \mathrm{eV}$. At the upper boundary of lower mantle convection zone it is set equal to 5.6 $\mathrm{eV}$. The lower (upper) boundary of the upper (lower) mantle convection cell is assumed to be at depths between 500 and 700 $\mathrm{km}$. The lower boundary of the lower mantle convection is assumed to be at the core-mantle boundary.

\section{§3. Results and Conclusions}

The parameters $\left(U B, d, T_{U B}\right.$, and $T_{L B}$ ) adopted in the calculations and the physical values $\left(\eta, R a, N u\right.$, and $\left.T_{m}\right)$ obtained from the calculations are illustrated in Tables 1 , $2,3,4,5$, and 6 and Figures 2, 3, 4, 5, and 6. The blank space in the table shows that convection current is absent from the model. In the calculation of Rayleigh number the viscosity is set equal to its value at the upper boundary of the convecting region.

Main results are:

1. The temperature at the bottom of upper mantle convection cell is about 1450$1650^{\circ} \mathrm{K}$ regardless of the conduction equation

\begin{tabular}{|c|c|c|c|c|c|c|c|c|}
\hline Model & $\underset{\mathrm{km}}{U B, *}$ & $\underset{\mathrm{km}}{d}$ & $T_{{ }^{\circ} \mathrm{K}}$, & $\begin{array}{c}T_{L B}, * * \\
{ }^{\circ} \mathrm{K}\end{array}$ & $\begin{array}{c}\eta \\
\text { poise }\end{array}$ & $R a$ & $N u$ & $T_{\circ}$, \\
\hline $0-500^{\prime}$ & $\begin{array}{r}50 \\
500\end{array}$ & $\begin{array}{r}450 \\
2400\end{array}$ & $\begin{array}{l}1200 \\
2055\end{array}$ & $\begin{array}{l}2055 \\
3280\end{array}$ & $\begin{array}{l}5.36 \times 10^{22} \\
6.58 \times 10^{24}\end{array}$ & $\begin{array}{l}7.06 \times 10^{3} \\
1.68 \times 10^{4}\end{array}$ & $\begin{array}{l}2.83 \\
4.07\end{array}$ & $\begin{array}{l}1502 \\
2356\end{array}$ \\
\hline $0-500$ & $\begin{array}{r}80 \\
500\end{array}$ & $\begin{array}{r}420 \\
2400\end{array}$ & $\begin{array}{l}1420 \\
2055\end{array}$ & $\begin{array}{l}2055 \\
3280\end{array}$ & $\begin{array}{l}1.58 \times 10^{20} \\
1.09 \times 10^{25}\end{array}$ & $\begin{array}{l}1.17 \times 10^{6} \\
7.83 \times 10^{3}\end{array}$ & $\begin{array}{l}9.97 \\
3.02\end{array}$ & $\begin{array}{l}1484 \\
2463\end{array}$ \\
\hline $0-500^{\prime \prime}$ & $\begin{array}{l}110 \\
500\end{array}$ & $\begin{array}{r}390 \\
2400\end{array}$ & $\begin{array}{l}1520 \\
2055\end{array}$ & $\begin{array}{l}2055 \\
3280\end{array}$ & $\begin{array}{l}1.92 \times 10^{19} \\
1.48 \times 10^{24}\end{array}$ & $\begin{array}{l}6.05 \times 10^{6} \\
5.24 \times 10^{4}\end{array}$ & $\begin{array}{r}14.39 \\
4.95\end{array}$ & $\begin{array}{l}1557 \\
2302\end{array}$ \\
\hline $0-600^{\prime}$ & $\begin{array}{r}50 \\
600\end{array}$ & $\begin{array}{r}550 \\
2300\end{array}$ & $\begin{array}{l}1200 \\
2148\end{array}$ & $\begin{array}{l}2143 \\
3280\end{array}$ & $\begin{array}{l}5.36 \times 10^{22} \\
3.28 \times 10^{25}\end{array}$ & $\begin{array}{l}1.42 \times 10^{4} \\
2.55 \times 10^{3}\end{array}$ & 3.81 & 1447 \\
\hline $0-600$ & $\begin{array}{r}80 \\
600\end{array}$ & $\begin{array}{r}520 \\
2300\end{array}$ & $\begin{array}{l}1420 \\
2143\end{array}$ & $\begin{array}{l}2143 \\
3280\end{array}$ & $\begin{array}{l}1.58 \times 10^{20} \\
1.16 \times 10^{25}\end{array}$ & $\begin{array}{l}2.43 \times 10^{6} \\
6.86 \times 10^{3}\end{array}$ & $\begin{array}{r}11.75 \\
2.92\end{array}$ & $\begin{array}{l}1482 \\
2529\end{array}$ \\
\hline $0-600^{\prime \prime}$ & $\begin{array}{l}110 \\
600\end{array}$ & $\begin{array}{r}490 \\
2300\end{array}$ & $\begin{array}{l}1520 \\
2143\end{array}$ & $\begin{array}{l}2143 \\
3280\end{array}$ & $\begin{array}{l}1.92 \times 10^{19} \\
1.51 \times 10^{24}\end{array}$ & $\begin{array}{l}1.39 \times 10^{7} \\
4.71 \times 10^{4}\end{array}$ & $\begin{array}{r}17.40 \\
4.83\end{array}$ & $\begin{array}{l}1556 \\
2378\end{array}$ \\
\hline $0-700^{\prime}$ & $\begin{array}{r}50 \\
700\end{array}$ & $\begin{array}{r}650 \\
2200\end{array}$ & $\begin{array}{l}1200 \\
2221\end{array}$ & $\begin{array}{l}2221 \\
3280\end{array}$ & $\begin{array}{l}5.36 \times 10^{22} \\
8.07 \times 10^{25}\end{array}$ & $\begin{array}{l}2.54 \times 10^{4} \\
9.71 \times 10^{2}\end{array}$ & 4.67 & 1418 \\
\hline $0-700$ & $\begin{array}{r}80 \\
700\end{array}$ & $\begin{array}{r}620 \\
2200\end{array}$ & $\begin{array}{l}1420 \\
2221\end{array}$ & $\begin{array}{l}2221 \\
3280\end{array}$ & $\begin{array}{l}1.58 \times 10^{20} \\
1.34 \times 10^{25}\end{array}$ & $\begin{array}{l}4.74 \times 10^{6} \\
5.31 \times 10^{3}\end{array}$ & $\begin{array}{r}13.64 \\
2.51\end{array}$ & $\begin{array}{l}1477 \\
2642\end{array}$ \\
\hline $0-700^{\prime \prime}$ & $\begin{array}{l}110 \\
700\end{array}$ & $\begin{array}{r}590 \\
2200\end{array}$ & $\begin{array}{l}1520 \\
2221\end{array}$ & $\begin{array}{l}2221 \\
3280\end{array}$ & $\begin{array}{l}1.92 \times 10^{19} \\
1.55 \times 10^{24}\end{array}$ & $\begin{array}{l}2.81 \times 10^{7} \\
4.11 \times 10^{4}\end{array}$ & $\begin{array}{r}20.38 \\
4.69\end{array}$ & $\begin{array}{l}1555 \\
2447\end{array}$ \\
\hline
\end{tabular}

Table 1. Data for oceanic mantle (model OCEAN).

* $U B$; the depth of the upper boundary of fluid layer.

** $T_{L B}$; the temperature at the lower boundary of the fluid layer calculated from conduction theory. 
Table 2. Data for shield mantle model (model SHIELD).

\begin{tabular}{|c|c|c|c|c|c|c|c|c|}
\hline Model & $\begin{array}{l}U B \\
\mathrm{~km}\end{array}$ & $\underset{\mathrm{km}}{d,}$ & $T_{{ }^{\circ B}}$ & ${ }^{T_{L B} \mathrm{~K}}$ & $\begin{array}{c}\eta \\
\text { poise }\end{array}$ & $R a$ & $N u$ & $\stackrel{T_{m}}{{ }^{\circ}}$ \\
\hline S-500' & $\begin{array}{l}120 \\
500\end{array}$ & $\begin{array}{r}380 \\
2400\end{array}$ & $\begin{array}{l}1285 \\
1990\end{array}$ & $\begin{array}{l}1990 \\
3270\end{array}$ & $\begin{array}{l}4.44 \times 10^{21} \\
5.71 \times 10^{25}\end{array}$ & $\begin{array}{l}4.53 \times 10^{4} \\
2.03 \times 10^{3}\end{array}$ & 4.89 & 1429 \\
\hline$S-500$ & $\begin{array}{l}155 \\
500\end{array}$ & $\begin{array}{r}345 \\
2400\end{array}$ & $\begin{array}{l}1396 \\
1990\end{array}$ & $\begin{array}{l}1990 \\
3270\end{array}$ & $\begin{array}{l}2.73 \times 10^{20} \\
1.56 \times 10^{25}\end{array}$ & $\begin{array}{l}3.99 \times 10^{5} \\
5.89 \times 10^{3}\end{array}$ & $\begin{array}{l}7.82 \\
2.58\end{array}$ & $\begin{array}{l}1472 \\
2486\end{array}$ \\
\hline $\mathrm{S}-500^{\prime \prime}$ & $\begin{array}{l}180 \\
500\end{array}$ & $\begin{array}{r}320 \\
2400\end{array}$ & $\begin{array}{l}1475 \\
1990\end{array}$ & $\begin{array}{l}1990 \\
3270\end{array}$ & $\begin{array}{l}4.85 \times 10^{19} \\
3.47 \times 10^{24}\end{array}$ & $\begin{array}{l}1.41 \times 10^{6} \\
2.44 \times 10^{4}\end{array}$ & $\begin{array}{r}10.39 \\
4.61\end{array}$ & $\begin{array}{l}1525 \\
2267\end{array}$ \\
\hline$S-600^{\prime}$ & $\begin{array}{l}120 \\
600\end{array}$ & $\begin{array}{r}480 \\
2300\end{array}$ & $\begin{array}{l}1285 \\
2085\end{array}$ & $\begin{array}{l}2085 \\
3270\end{array}$ & $\begin{array}{l}4.44 \times 10^{21} \\
6.89 \times 10^{25}\end{array}$ & $\begin{array}{l}1.03 \times 10^{5} \\
1.31 \times 10^{3}\end{array}$ & 5.77 & 1423 \\
\hline$S-600$ & $\begin{array}{l}155 \\
600\end{array}$ & $\begin{array}{r}445 \\
2300\end{array}$ & $\begin{array}{l}1396 \\
2085\end{array}$ & $\begin{array}{l}2085 \\
3270\end{array}$ & $\begin{array}{l}2.73 \times 10^{20} \\
1.60 \times 10^{25}\end{array}$ & $\begin{array}{l}1.00 \times 10^{6} \\
5.23 \times 10^{3}\end{array}$ & $\begin{array}{l}9.26 \\
2.42\end{array}$ & $\begin{array}{l}1471 \\
2576\end{array}$ \\
\hline S-600" & $\begin{array}{l}180 \\
600\end{array}$ & $\begin{array}{r}420 \\
2300\end{array}$ & $\begin{array}{l}1475 \\
2085\end{array}$ & $\begin{array}{l}2085 \\
3270\end{array}$ & $\begin{array}{l}4.85 \times 10^{19} \\
3.12 \times 10^{24}\end{array}$ & $\begin{array}{l}2.06 \times 10^{6} \\
2.48 \times 10^{4}\end{array}$ & $\begin{array}{r}11.32 \\
4.65\end{array}$ & $\begin{array}{l}1529 \\
2340\end{array}$ \\
\hline$S-700^{\prime}$ & $\begin{array}{l}120 \\
700\end{array}$ & $\begin{array}{r}580 \\
2200\end{array}$ & $\begin{array}{l}1285 \\
2173\end{array}$ & $\begin{array}{l}2173 \\
3270\end{array}$ & $\begin{array}{l}4.44 \times 10^{21} \\
8.33 \times 10^{25}\end{array}$ & $\begin{array}{l}2.03 \times 10^{5} \\
9.76 \times 10^{2}\end{array}$ & 6.71 & 1417 \\
\hline$S-700$ & $\begin{array}{l}155 \\
700\end{array}$ & $\begin{array}{r}545 \\
2200\end{array}$ & $\begin{array}{l}1396 \\
2173\end{array}$ & $\begin{array}{l}2173 \\
3270\end{array}$ & $\begin{array}{l}2.73 \times 10^{20} \\
3.48 \times 10^{24}\end{array}$ & $\begin{array}{l}1.26 \times 10^{5} \\
2.04 \times 10^{4}\end{array}$ & $\begin{array}{l}6.04 \\
4.01\end{array}$ & $\begin{array}{l}1525 \\
2455\end{array}$ \\
\hline$S-700^{\prime \prime}$ & $\begin{array}{l}180 \\
700\end{array}$ & $\begin{array}{r}520 \\
2200\end{array}$ & $\begin{array}{l}1475 \\
2173\end{array}$ & $\begin{array}{l}2173 \\
3270\end{array}$ & $\begin{array}{l}4.85 \times 10^{19} \\
3.99 \times 10^{24}\end{array}$ & $\begin{array}{l}8.22 \times 10^{6} \\
1.75 \times 10^{4}\end{array}$ & $\begin{array}{r}15.45 \\
4.11\end{array}$ & $\begin{array}{l}1520 \\
2440\end{array}$ \\
\hline
\end{tabular}

Table 3. Data for hot model (model $\mathrm{H} 1$ ).

\begin{tabular}{|c|c|c|c|c|c|c|c|c|}
\hline Model & $\begin{array}{l}U B \\
\mathrm{~km}\end{array}$ & $\underset{\mathrm{km}}{d,}$ & $\begin{array}{c}T_{U B}, \\
{ }^{\circ} \mathrm{K}\end{array}$ & $T_{{ }^{L B B}}$ & poise & $R a$ & $N u$ & $\stackrel{T}{m}_{\mathrm{K}}$ \\
\hline $\mathrm{H} 1-500^{\prime}$ & $\begin{array}{r}40 \\
500\end{array}$ & $\begin{array}{r}460 \\
2400\end{array}$ & $\begin{array}{l}1050 \\
3150\end{array}$ & $\begin{array}{l}3150 \\
4070\end{array}$ & $1.18 \times 10^{25}$ & $1.00 \times 10^{2}$ & & \\
\hline $\mathrm{H} 1-500$ & $\begin{array}{r}60 \\
500\end{array}$ & $\begin{array}{r}440 \\
2400\end{array}$ & $\begin{array}{l}1400 \\
3150\end{array}$ & $\begin{array}{l}3150 \\
4070\end{array}$ & $\begin{array}{l}2.48 \times 10^{20} \\
1.68 \times 10^{24}\end{array}$ & $\begin{array}{l}2.23 \times 10^{6} \\
3.48 \times 10^{4}\end{array}$ & $\begin{array}{r}11.52 \\
4.51\end{array}$ & $\begin{array}{l}1552 \\
3354\end{array}$ \\
\hline $\mathrm{H} 1-500^{\prime \prime}$ & $\begin{array}{r}70 \\
500\end{array}$ & $\begin{array}{r}430 \\
2400\end{array}$ & $\begin{array}{l}1610 \\
3150\end{array}$ & $\begin{array}{l}3150 \\
4070\end{array}$ & $\begin{array}{l}8.12 \times 10^{18} \\
7.67 \times 10^{22}\end{array}$ & $\begin{array}{l}4.33 \times 10^{7} \\
6.37 \times 10^{5}\end{array}$ & $\begin{array}{r}22.45 \\
8.69\end{array}$ & $\begin{array}{l}1679 \\
3256\end{array}$ \\
\hline $\mathrm{H} 1-600^{\prime}$ & $\begin{array}{r}40 \\
600\end{array}$ & $\begin{array}{r}560 \\
2300\end{array}$ & $\begin{array}{l}1050 \\
3260\end{array}$ & $\begin{array}{l}3260 \\
4070\end{array}$ & $\begin{array}{l}1.18 \times 10^{25} \\
3.87 \times 10^{23}\end{array}$ & $\begin{array}{l}1.90 \times 10^{4} \\
1.21 \times 10^{5}\end{array}$ & $\begin{array}{l}3.94 \\
5.99\end{array}$ & $\begin{array}{l}1610 \\
3395\end{array}$ \\
\hline $\mathrm{H} 1-600$ & $\begin{array}{r}60 \\
600\end{array}$ & $\begin{array}{r}540 \\
2300\end{array}$ & $\begin{array}{l}1390 \\
3260\end{array}$ & $\begin{array}{l}3260 \\
4070\end{array}$ & $\begin{array}{l}3.13 \times 10^{20} \\
2.58 \times 10^{24}\end{array}$ & $\begin{array}{l}3.55 \times 10^{6} \\
1.88 \times 10^{4}\end{array}$ & $\begin{array}{r}12.79 \\
3.93\end{array}$ & $\begin{array}{l}1536 \\
3466\end{array}$ \\
\hline $\mathrm{H} 1-600^{\prime \prime}$ & $\begin{array}{r}80 \\
600\end{array}$ & $\begin{array}{r}520 \\
2300\end{array}$ & $\begin{array}{l}1790 \\
3260\end{array}$ & $\begin{array}{l}3260 \\
4070\end{array}$ & $\begin{array}{l}2.31 \times 10^{17} \\
4.31 \times 10^{21}\end{array}$ & $\begin{array}{l}1.98 \times 10^{9} \\
8.18 \times 10^{6}\end{array}$ & $\begin{array}{l}53.07 \\
15.43\end{array}$ & $\begin{array}{l}1818 \\
3312\end{array}$ \\
\hline $\mathrm{H} 1-700^{\prime}$ & $\begin{array}{r}40 \\
700\end{array}$ & $\begin{array}{r}660 \\
2200\end{array}$ & $\begin{array}{l}1050 \\
3345\end{array}$ & $\begin{array}{l}3345 \\
4070\end{array}$ & $1.18 \times 10^{25}$ & $3.23 \times 10^{2}$ & & \\
\hline H1-700 & $\begin{array}{r}60 \\
700\end{array}$ & $\begin{array}{r}640 \\
2200\end{array}$ & $\begin{array}{l}1400 \\
3345\end{array}$ & $\begin{array}{l}3345 \\
4070\end{array}$ & $\begin{array}{l}2.48 \times 10^{20} \\
3.20 \times 10^{24}\end{array}$ & $\begin{array}{l}7.65 \times 10^{6} \\
1.42 \times 10^{4}\end{array}$ & $\begin{array}{r}15.20 \\
3.67\end{array}$ & $\begin{array}{l}1528 \\
3542\end{array}$ \\
\hline $\mathrm{H} 1-700^{\prime \prime}$ & $\begin{array}{r}80 \\
700\end{array}$ & $\begin{array}{r}620 \\
2200\end{array}$ & $\begin{array}{l}1790 \\
3345\end{array}$ & $\begin{array}{l}3345 \\
4070\end{array}$ & $\begin{array}{l}2.31 \times 10^{17} \\
4.56 \times 10^{21}\end{array}$ & $\begin{array}{l}3.71 \times 10^{9} \\
6.67 \times 10^{6}\end{array}$ & $\begin{array}{l}61.11 \\
14.74\end{array}$ & $\begin{array}{l}1815 \\
3394\end{array}$ \\
\hline
\end{tabular}

solution and the thickness of the convecting layer.

2. If the temperature at the bottom of upper mantle convection cell is smaller than about $1450^{\circ} \mathrm{K}$, then the lower mantle convection does not occur.

3. Variation of depth of the lower boundary of the upper mantle convection between
500 and $700 \mathrm{~km}$ affects little on the temperature within the convecting region of the upper mantle. In this case, the temperature at the lower boundary of lower mantle convection changes by some 300 degrees.

4. If the convection is present, then the temperature gradient within the upper mantle differs very little from the adiabatic gradient. 
Table 4. Data for cold model (model L1).

\begin{tabular}{|c|c|c|c|c|c|c|c|c|}
\hline Model & $\begin{array}{l}U B \\
\mathrm{~km}\end{array}$ & $\underset{\mathrm{km}}{d,}$ & $\begin{array}{l}T_{U B}, \\
{ }^{\circ} \mathrm{K}\end{array}$ & ${ }_{{ }^{\circ} \mathrm{K}}$ & $\begin{array}{c}\eta \\
\text { poise }\end{array}$ & $R a$ & $N u$ & ${ }^{T_{m}}{ }_{\mathrm{K}}$, \\
\hline L1-500' & $\begin{array}{l}250 \\
500\end{array}$ & $\begin{array}{r}250 \\
2400\end{array}$ & $\begin{array}{l}1228 \\
1505\end{array}$ & $\begin{array}{l}1505 \\
2808\end{array}$ & $2.27 \times 10^{22}$ & $1.15 \times 10^{3}$ & & \\
\hline L1-500 & $\begin{array}{l}290 \\
500\end{array}$ & $\begin{array}{r}210 \\
2400\end{array}$ & $\begin{array}{l}1280 \\
1505\end{array}$ & $\begin{array}{l}1505 \\
2808\end{array}$ & $\begin{array}{l}5.09 \times 10^{21} \\
3.18 \times 10^{25}\end{array}$ & $\begin{array}{l}2.33 \times 10^{3} \\
3.03 \times 10^{3}\end{array}$ & $\begin{array}{l}1.33 \\
1.79\end{array}$ & $\begin{array}{l}1448 \\
2232\end{array}$ \\
\hline $\mathrm{L} 1-500^{\prime \prime}$ & $\begin{array}{l}330 \\
500\end{array}$ & $\begin{array}{r}170 \\
2400\end{array}$ & $\begin{array}{l}1330 \\
1505\end{array}$ & $\begin{array}{l}1505 \\
2808\end{array}$ & $\begin{array}{l}1.35 \times 10^{21} \\
6.89 \times 10^{25}\end{array}$ & $\begin{array}{l}3.42 \times 10^{3} \\
1.45 \times 10^{3}\end{array}$ & 1.87 & 1423 \\
\hline $\mathrm{L} 1-600^{\prime}$ & $\begin{array}{l}220 \\
600\end{array}$ & $\begin{array}{r}380 \\
2300\end{array}$ & $\begin{array}{l}1183 \\
1593\end{array}$ & $\begin{array}{l}1593 \\
2808\end{array}$ & $9.21 \times 10^{22}$ & $1.55 \times 10^{3}$ & & \\
\hline LI-600 & $\begin{array}{l}230 \\
600\end{array}$ & $\begin{array}{r}370 \\
2300\end{array}$ & $\begin{array}{l}1200 \\
1593\end{array}$ & $\begin{array}{l}1593 \\
2808\end{array}$ & $\begin{array}{l}5.36 \times 10^{22} \\
1.27 \times 10^{25}\end{array}$ & $\begin{array}{l}2.31 \times 10^{3} \\
6.70 \times 10^{3}\end{array}$ & $\begin{array}{l}1.40 \\
2.84\end{array}$ & $\begin{array}{l}1480 \\
2021\end{array}$ \\
\hline$L 1-600^{\prime \prime}$ & $\begin{array}{l}240 \\
600\end{array}$ & $\begin{array}{r}360 \\
2300\end{array}$ & $\begin{array}{l}1213 \\
1593\end{array}$ & $\begin{array}{l}1593 \\
2808\end{array}$ & $\begin{array}{l}3.58 \times 10^{22} \\
6.47 \times 10^{25}\end{array}$ & $\begin{array}{l}3.04 \times 10^{3} \\
1.43 \times 10^{3}\end{array}$ & 1.79 & 1425 \\
\hline $\mathrm{L} 1-700^{\prime}$ & $\begin{array}{l}200 \\
700\end{array}$ & $\begin{array}{r}500 \\
2200\end{array}$ & $\begin{array}{l}1150 \\
1673\end{array}$ & $\begin{array}{l}1673 \\
2808\end{array}$ & $2.76 \times 10^{23}$ & $1.56 \times 10^{3}$ & & \\
\hline L1-700 & $\begin{array}{l}210 \\
700\end{array}$ & $\begin{array}{r}490 \\
2200\end{array}$ & $\begin{array}{l}1170 \\
1673\end{array}$ & $\begin{array}{l}1673 \\
2808\end{array}$ & $\begin{array}{l}1.41 \times 10^{23} \\
1.51 \times 10^{25}\end{array}$ & $\begin{array}{l}2.70 \times 10^{3} \\
5.12 \times 10^{3}\end{array}$ & $\begin{array}{l}1.66 \\
2.43\end{array}$ & $\begin{array}{l}1473 \\
2139\end{array}$ \\
\hline L1-700" & $\begin{array}{l}220 \\
700\end{array}$ & $\begin{array}{r}480 \\
2200\end{array}$ & $\begin{array}{l}1183 \\
1673\end{array}$ & $\begin{array}{l}1673 \\
2808\end{array}$ & $9.21 \times 10^{22}$ & $3.74 \times 10^{3}$ & 2.09 & 1417 \\
\hline
\end{tabular}

Table 5. Data for hot model (model H2).

\begin{tabular}{|c|c|c|c|c|c|c|c|c|}
\hline Model & $\begin{array}{l}U B \\
\mathrm{~km}\end{array}$ & $\begin{array}{c}d, \\
\mathrm{~km}\end{array}$ & $T_{{ }^{\circ K} \mathrm{~K}}$ & $\begin{array}{l}T_{L B}, \\
{ }^{\circ} \mathrm{K}\end{array}$ & $\begin{array}{c}\eta \\
\text { poise }\end{array}$ & $R a$ & $N u$ & ${ }_{{ }^{\circ} \mathrm{K}}^{T_{\mathrm{K}}}$ \\
\hline $\mathrm{H} 2-500^{\prime}$ & $\begin{array}{r}50 \\
500\end{array}$ & $\begin{array}{r}450 \\
2400\end{array}$ & $\begin{array}{l}1280 \\
3545\end{array}$ & $\begin{array}{l}3545 \\
5250\end{array}$ & $\begin{array}{l}5.09 \times 10^{21} \\
2.44 \times 10^{23}\end{array}$ & $\begin{array}{l}1.74 \times 10^{5} \\
3.98 \times 10^{5}\end{array}$ & $\begin{array}{l}6.49 \\
7.81\end{array}$ & $\begin{array}{l}1629 \\
3763\end{array}$ \\
\hline $\mathrm{H} 2-500$ & $\begin{array}{r}60 \\
500\end{array}$ & $\begin{array}{r}440 \\
2400\end{array}$ & $\begin{array}{l}1500 \\
3545\end{array}$ & $\begin{array}{l}3545 \\
5250\end{array}$ & $\begin{array}{l}2.91 \times 10^{19} \\
3.96 \times 10^{23}\end{array}$ & $\begin{array}{l}1.92 \times 10^{7} \\
2.52 \times 10^{5}\end{array}$ & $\begin{array}{r}18.70 \\
7.05\end{array}$ & $\begin{array}{l}1609 \\
3787\end{array}$ \\
\hline $\mathrm{H} 2-500^{\prime \prime}$ & $\begin{array}{r}65 \\
500\end{array}$ & $\begin{array}{r}435 \\
2400\end{array}$ & $\begin{array}{l}1600 \\
3545\end{array}$ & $\begin{array}{l}3545 \\
5250\end{array}$ & $\begin{array}{l}4.49 \times 10^{18} \\
5.03 \times 10^{22}\end{array}$ & $\begin{array}{l}1.59 \times 10^{8} \\
1.75 \times 10^{6}\end{array}$ & $\begin{array}{l}30.10 \\
10.91\end{array}$ & $\begin{array}{l}1698 \\
3701\end{array}$ \\
\hline $\mathrm{H} 2-600^{\prime}$ & $\begin{array}{r}50 \\
600\end{array}$ & $\begin{array}{r}550 \\
2300\end{array}$ & $\begin{array}{l}1280 \\
3690\end{array}$ & $\begin{array}{l}3690 \\
5250\end{array}$ & $\begin{array}{l}5.09 \times 10^{21} \\
5.07 \times 10^{23}\end{array}$ & $\begin{array}{l}3.40 \times 10^{5} \\
1.81 \times 10^{5}\end{array}$ & $\begin{array}{l}7.54 \\
6.55\end{array}$ & $\begin{array}{l}1599 \\
3928\end{array}$ \\
\hline $\mathrm{H} 2-600$ & $\begin{array}{r}60 \\
600\end{array}$ & $\begin{array}{r}540 \\
2300\end{array}$ & $\begin{array}{l}1500 \\
3690\end{array}$ & $\begin{array}{l}3690 \\
5250\end{array}$ & $\begin{array}{l}2.91 \times 10^{19} \\
4.95 \times 10^{23}\end{array}$ & $\begin{array}{l}3.80 \times 10^{7} \\
1.86 \times 10^{5}\end{array}$ & $\begin{array}{r}21.81 \\
6.58\end{array}$ & $\begin{array}{l}1600 \\
3927\end{array}$ \\
\hline $\mathrm{H} 2-600^{\prime \prime}$ & $\begin{array}{r}65 \\
600\end{array}$ & $\begin{array}{r}535 \\
2300\end{array}$ & $\begin{array}{l}1600 \\
3690\end{array}$ & $\begin{array}{l}3690 \\
5250\end{array}$ & $\begin{array}{l}4.49 \times 10^{18} \\
1.05 \times 10^{23}\end{array}$ & $\begin{array}{l}2.10 \times 10^{8} \\
7.96 \times 10^{5}\end{array}$ & $\begin{array}{r}32.05 \\
9.13\end{array}$ & $\begin{array}{l}1665 \\
3861\end{array}$ \\
\hline $\mathrm{H} 2-700^{\prime}$ & $\begin{array}{r}50 \\
700\end{array}$ & $\begin{array}{r}650 \\
2200\end{array}$ & $\begin{array}{l}1280 \\
3815\end{array}$ & $\begin{array}{l}3815 \\
5250\end{array}$ & $\begin{array}{l}5.09 \times 10^{21} \\
4.37 \times 10^{23}\end{array}$ & $\begin{array}{l}5.91 \times 10^{5} \\
1.85 \times 10^{5}\end{array}$ & $\begin{array}{l}7.78 \\
6.57\end{array}$ & $\begin{array}{l}1605 \\
4033\end{array}$ \\
\hline $\mathrm{H} 2-700$ & $\begin{array}{r}60 \\
700\end{array}$ & $\begin{array}{r}640 \\
2200\end{array}$ & $\begin{array}{l}1500 \\
3815\end{array}$ & $\begin{array}{l}3815 \\
5250\end{array}$ & $\begin{array}{l}2.91 \times 10^{19} \\
5.74 \times 10^{23}\end{array}$ & $\begin{array}{l}6.70 \times 10^{7} \\
1.43 \times 10^{5}\end{array}$ & $\begin{array}{r}24.66 \\
6.20\end{array}$ & $\begin{array}{l}1594 \\
4046\end{array}$ \\
\hline $\mathrm{H} 2-700^{\prime \prime}$ & $\begin{array}{r}65 \\
700\end{array}$ & $\begin{array}{r}635 \\
2200\end{array}$ & $\begin{array}{l}1600 \\
3815\end{array}$ & $\begin{array}{l}3815 \\
5250\end{array}$ & $\begin{array}{l}4.49 \times 10^{18} \\
1.15 \times 10^{23}\end{array}$ & $\begin{array}{l}3.73 \times 10^{8} \\
6.46 \times 10^{5}\end{array}$ & $\begin{array}{r}36.45 \\
8.71\end{array}$ & $\begin{array}{l}1661 \\
3980\end{array}$ \\
\hline
\end{tabular}

The temperature at the core-mantle boundary is about $3800^{\circ} \mathrm{K}$ for model $\mathrm{H} 2$ and $2100^{\circ} \mathrm{K}$ for model L1. The result is close to that given by LYUSTIKH (1969) by a quantitative study.

5. For model H1-600 the Rayleigh number within the convecting region of the upper mantle is $3.55 \times 10^{6}$ and that of the lower mantle is $1.88 \times 10^{4}$; for model $\mathrm{H} 2-600,3.80 \times$ $10^{7}$ and $1.86 \times 10^{5}$; for model S-600, $1.00 \times 10^{6}$ and $5.23 \times 10^{3}$; for model $0-600,2.43 \times 10^{6}$ and $6.86 \times 10^{3}$; for model L2-600, $4.47 \times 10^{3}$ and $3.92 \times 10^{3}$; and for model L1-600, $2.31 \times 10^{3}$ and $6.70 \times 10^{3}$, respectively. These show that the upper mantle of the earth is considerably less thermally stable than the lower mantle. 
Table 6. Data for cold model (model L2).

\begin{tabular}{|c|c|c|c|c|c|c|c|c|}
\hline Model & $\begin{array}{l}U B \\
\mathrm{~km}\end{array}$ & $\underset{\mathrm{km}}{d}$ & $T_{{ }^{\circ} \mathrm{K}}$ & $T_{L B}$ & $\begin{array}{c}\eta \\
\text { poise }\end{array}$ & $R a$ & $N u$ & ${ }^{T_{m}}$ \\
\hline L $2-500^{\prime}$ & $\begin{array}{l}150 \\
500\end{array}$ & $\begin{array}{r}350 \\
2400\end{array}$ & $\begin{array}{l}1190 \\
1680\end{array}$ & $\begin{array}{l}1680 \\
3060\end{array}$ & $7.36 \times 10^{22}$ & $1.73 \times 10^{3}$ & & \\
\hline L2-500 & $\begin{array}{l}160 \\
500\end{array}$ & $\begin{array}{r}340 \\
2400\end{array}$ & $\begin{array}{l}1210 \\
1680\end{array}$ & $\begin{array}{l}1680 \\
3060\end{array}$ & $\begin{array}{l}3.92 \times 10^{22} \\
7.80 \times 10^{24}\end{array}$ & $\begin{array}{l}2.79 \times 10^{3} \\
1.22 \times 10^{4}\end{array}$ & $\begin{array}{l}1.64 \\
3.57\end{array}$ & $\begin{array}{l}1496 \\
2066\end{array}$ \\
\hline $\mathrm{L} 2-500^{\prime \prime}$ & $\begin{array}{l}170 \\
500\end{array}$ & $\begin{array}{r}330 \\
2400\end{array}$ & $\begin{array}{l}1230 \\
1680\end{array}$ & $\begin{array}{l}1680 \\
3060\end{array}$ & $\begin{array}{l}2.14 \times 10^{22} \\
5.04 \times 10^{25}\end{array}$ & $\begin{array}{l}4.38 \times 10^{3} \\
2.07 \times 10^{3}\end{array}$ & 2.22 & 1433 \\
\hline $\mathrm{L} 2-600^{\prime}$ & $\begin{array}{l}120 \\
600\end{array}$ & $\begin{array}{r}480 \\
2300\end{array}$ & $\begin{array}{l}1110 \\
1785\end{array}$ & $\begin{array}{l}1785 \\
3060\end{array}$ & $1.14 \times 10^{24}$ & $4.38 \times 10^{2}$ & & \\
\hline L2-600 & $\begin{array}{l}150 \\
600\end{array}$ & $\begin{array}{r}450 \\
2300\end{array}$ & $\begin{array}{l}1190 \\
1785\end{array}$ & $\begin{array}{l}1785 \\
3060\end{array}$ & $\begin{array}{l}7.36 \times 10^{22} \\
2.35 \times 10^{25}\end{array}$ & $\begin{array}{l}4.47 \times 10^{3} \\
3.92 \times 10^{3}\end{array}$ & $\begin{array}{l}2.22 \\
2.15\end{array}$ & $\begin{array}{l}1458 \\
2378\end{array}$ \\
\hline $\mathrm{L} 2-600^{\prime \prime}$ & $\begin{array}{l}170 \\
600\end{array}$ & $\begin{array}{r}430 \\
2300\end{array}$ & $\begin{array}{l}1230 \\
1785\end{array}$ & $\begin{array}{l}1785 \\
3060\end{array}$ & $\begin{array}{l}2.14 \times 10^{22} \\
1.90 \times 10^{26}\end{array}$ & $1.20 \times 10^{4}$ & 3.41 & 1393 \\
\hline L2- $700^{\prime}$ & $\begin{array}{l}130 \\
700\end{array}$ & $\begin{array}{r}570 \\
2200\end{array}$ & $\begin{array}{l}1140 \\
1880\end{array}$ & $\begin{array}{l}1880 \\
3060\end{array}$ & $\begin{array}{l}3.90 \times 10^{23} \\
9.85 \times 10^{22}\end{array}$ & $\begin{array}{l}2.27 \times 10^{3} \\
6.18 \times 10^{5}\end{array}$ & $\begin{array}{l}1.40 \\
8.62\end{array}$ & $\begin{array}{l}1668 \\
2017\end{array}$ \\
\hline $\mathrm{L} 2-700$ & $\begin{array}{l}135 \\
700\end{array}$ & $\begin{array}{r}565 \\
2200\end{array}$ & $\begin{array}{l}1158 \\
1880\end{array}$ & $\begin{array}{l}1880 \\
3060\end{array}$ & $\begin{array}{l}2.10 \times 10^{23} \\
8.49 \times 10^{24}\end{array}$ & $\begin{array}{l}3.90 \times 10^{3} \\
9.21 \times 10^{3}\end{array}$ & $\begin{array}{l}2.15 \\
3.16\end{array}$ & $\begin{array}{l}1493 \\
2253\end{array}$ \\
\hline $\mathrm{L} 2-700^{\prime \prime}$ & $\begin{array}{l}140 \\
700\end{array}$ & $\begin{array}{r}560 \\
2200\end{array}$ & $\begin{array}{l}1170 \\
1880\end{array}$ & $\begin{array}{l}1880 \\
3060\end{array}$ & $\begin{array}{l}1.41 \times 10^{23} \\
3.18 \times 10^{25}\end{array}$ & $\begin{array}{l}5.50 \times 10^{3} \\
2.62 \times 10^{3}\end{array}$ & $\begin{array}{l}2.55 \\
1.63\end{array}$ & $\begin{array}{l}1448 \\
2602\end{array}$ \\
\hline
\end{tabular}

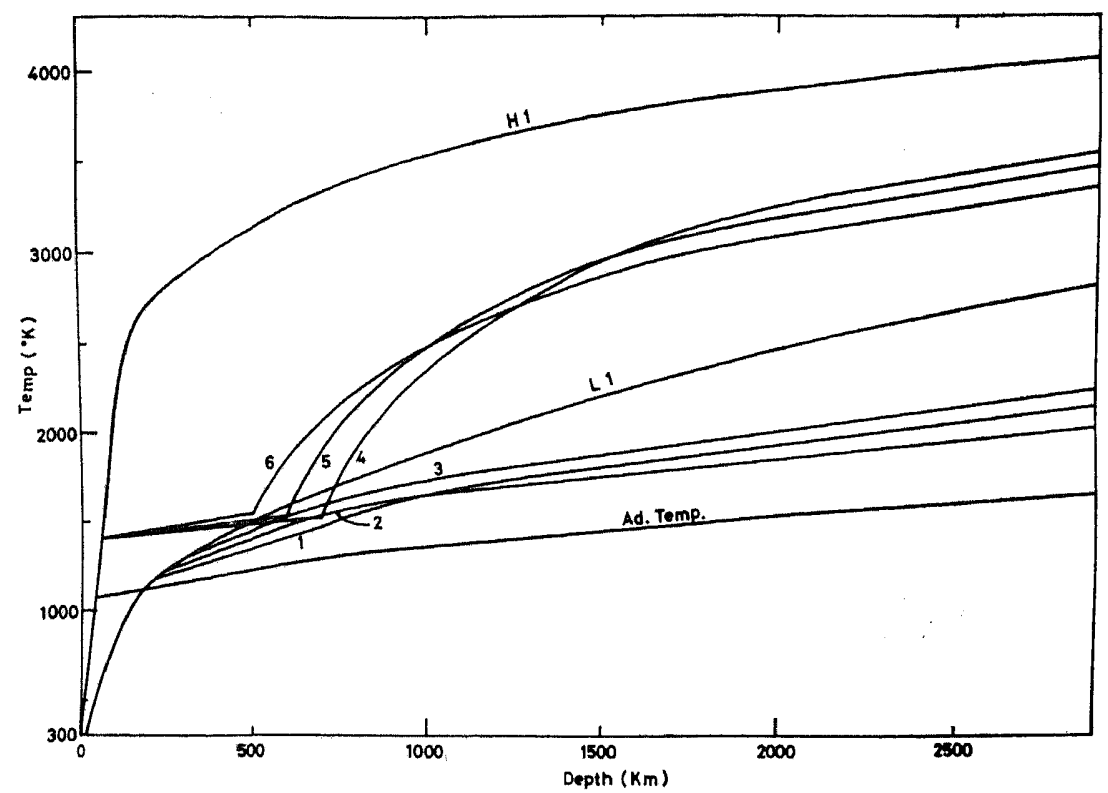

Fig. 2. Steady convection solutions $T(r)$ for hot (H1, IRIYAMA, 1970, his model RC-U2) and cold (L1) models.

H1: 4, 5 and 6; Upper boundary of the upper mantle convection at depth $60 \mathrm{~km}$. Upper boundary of the lower mantle convection at depth 700,600 and $500 \mathrm{~km}$, respectively.

L1: 1, 2 and 3; Upper boundary of the upper mantle convection at depth 210, 230 and $290 \mathrm{~km}$, respectively. Upper boundary of the lower mantle convection at depth 700,600 and $500 \mathrm{~km}$, respectively.

Ad. Temp.; Adiabatic temperature distribution by VERHOOGEN (1951). 


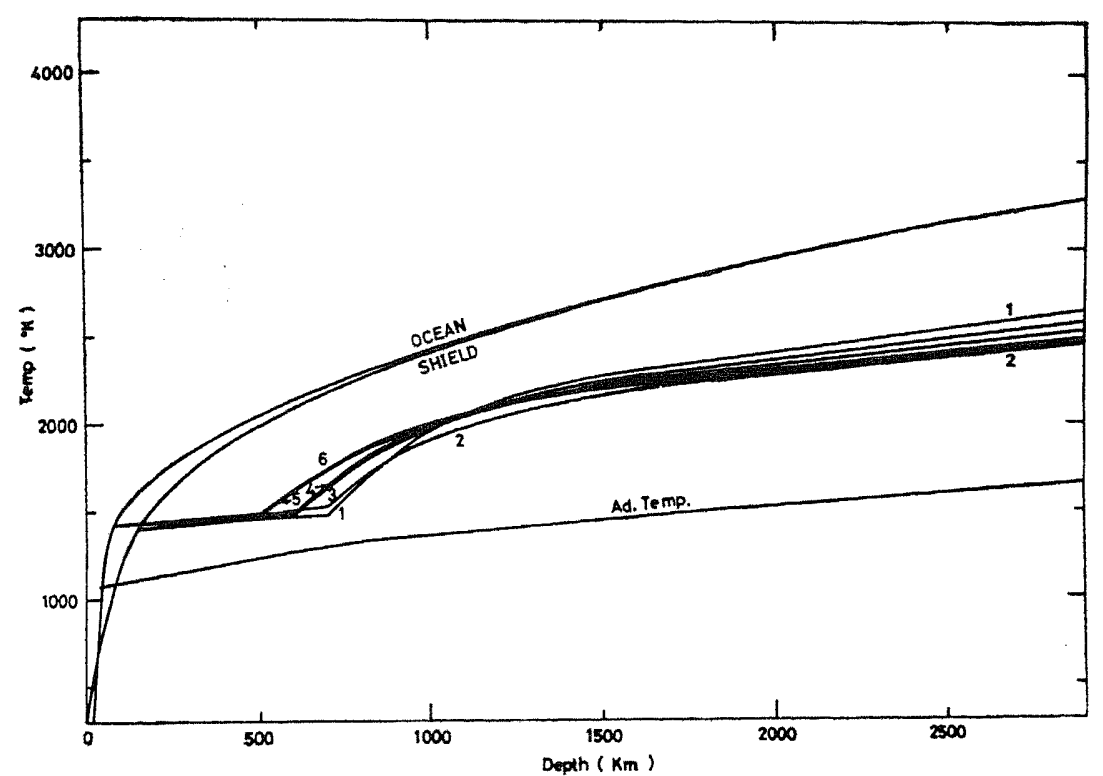

Fig. 3. Steady convection solutions $T(r)$ for oceanic and shield mantle models (from $0-400$ km depth, ClaAk and RINGWOOD, 1964).

OCEAN: 1, 4 and 6; Upper boundary of the upper mantle convection at depth $80 \mathrm{~km}$. Upper boundary of the lower mantle convection at depth 700,600 and $500 \mathrm{~km}$, respectively.

SHIELD: 2, 3 and 5; Upper boundary of the upper mantle convection at depth $155 \mathrm{~km}$. Upper boundary of the lower mantle convection at depth 700,600 and $500 \mathrm{~km}$, respectively.

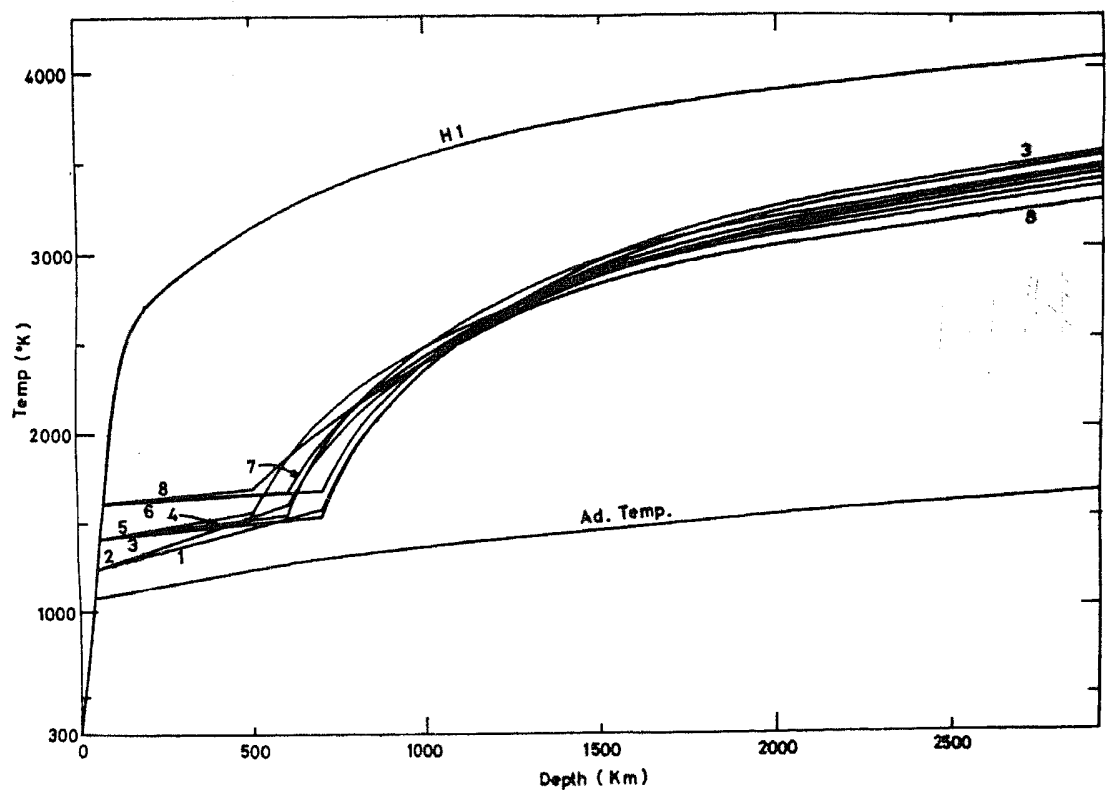

Fig. 4. Steady convection solutions $T(r)$ for model $\mathrm{H}$.

$1,2,3,4,5,6,7$ and 8; Upper boundary of the upper mantle convection at depth $50,50,60,60,60,70,70$ and $70 \mathrm{~km}$, respectively. Upper boundary of the lower mantle convection at depth $700,600,700,600,500,700,600$ and $500 \mathrm{~km}$, respectively. 


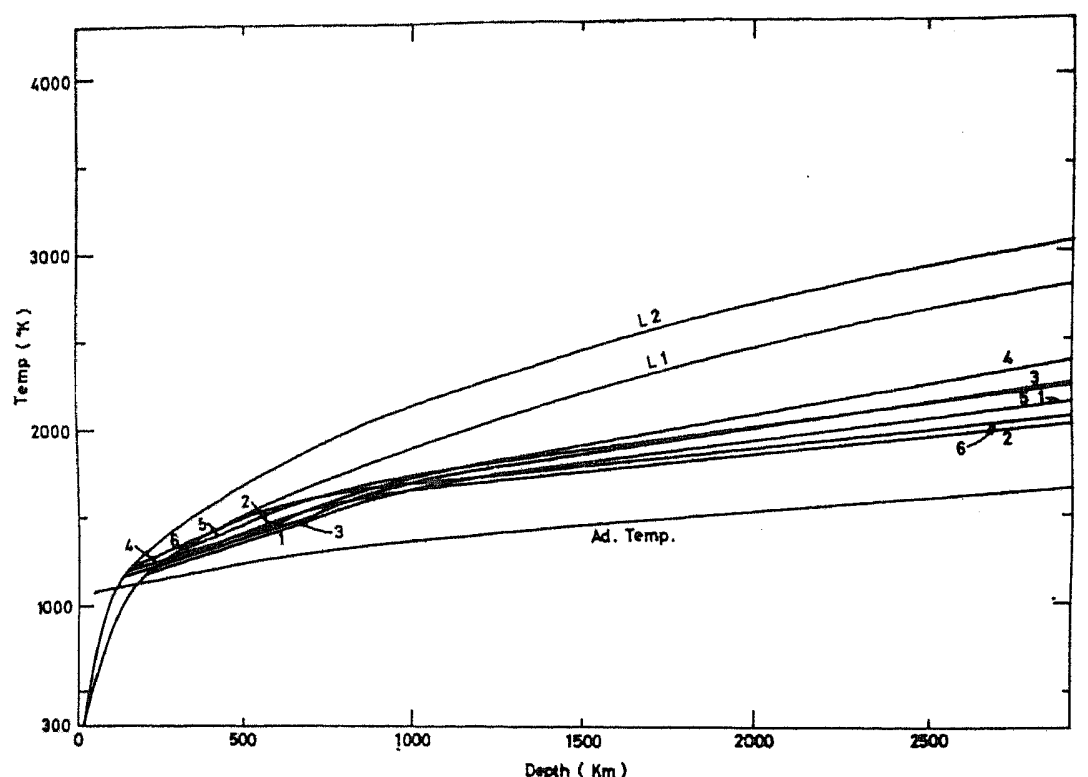

Fig. 5. Steady convection solutions $T(r)$ for cold models (L1 and L2).

L1: 1,2 and 5; Upper boundary of the upper mantle convection at depth 210, 230 and $290 \mathrm{~km}$, respectively. Upper boundary of the lower mantle convection at depth 700,600 and $500 \mathrm{~km}$, respectively.

L2: 3,4 and 6; Upper boundary of the upper mantle convection at depth 135,150 and $160 \mathrm{~km}$, respectively. Upper boundary of the lower mantle convection at depth 700,600 and $500 \mathrm{~km}$, respectively.

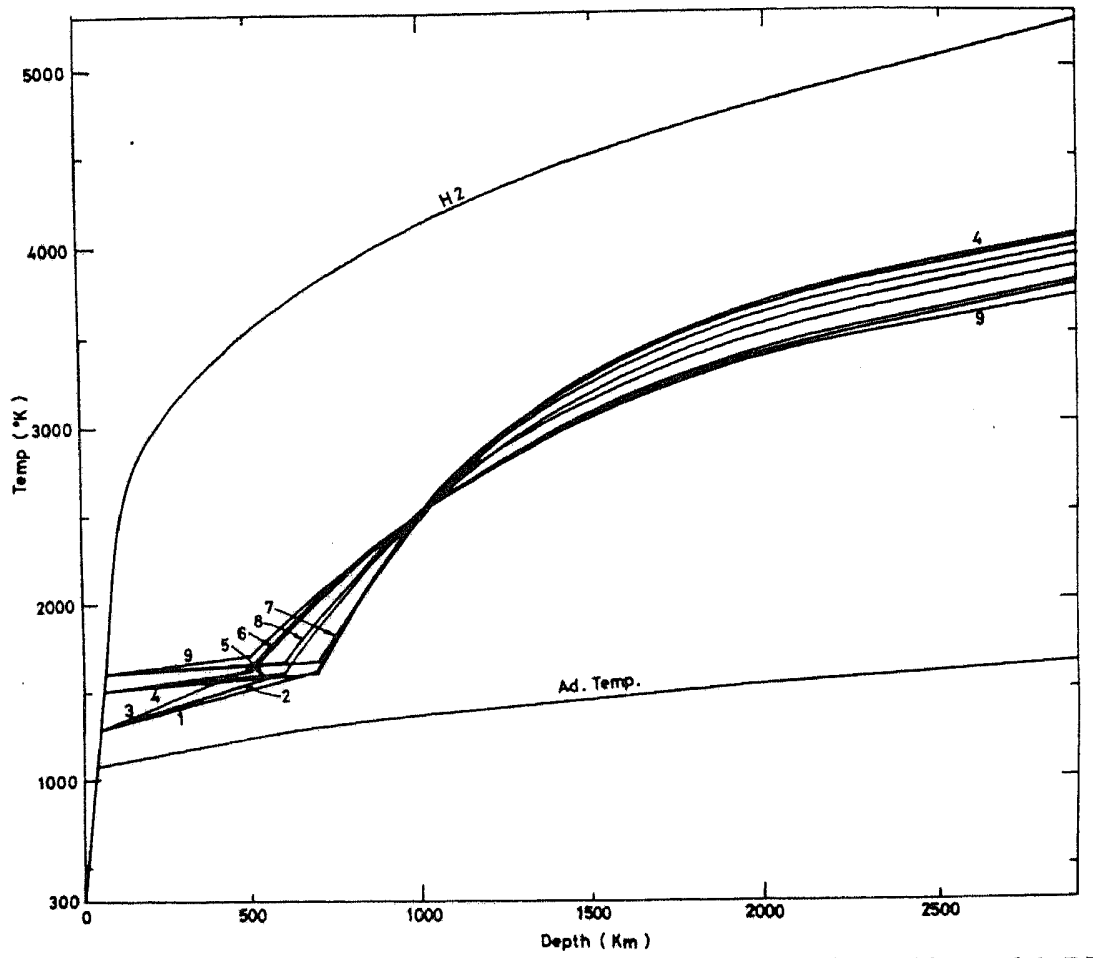

Fig. 6. Steady convection solutions $T(r)$ for hot (H2, IRIYAMA, 1970, his model RH-U2) model.

$1,2,3,4,5,6,7,8$ and 9 ; Upper boundary of the upper mantle convection at depth $50,50,50,60,60,60,65,65$ and $65 \mathrm{~km}$, respectively. Upper boundary of the lower mantle convection at depth $700,600,500,700,600,500,700,600$ and $500 \mathrm{~km}$, respectively. 
6. The thermal structure for the convecting zone under the oceans differs very little from that under the continents.

7. If the temperature at the bottom of the upper mantle convection cell is low, then the temperature of the lower mantle convection system is high. This results from the fact that the viscosity becomes larger or the Rayleigh number smaller as the temperature becomes lower.

\section{References}

Clark, S.P., and A.E. Ringwood, Density and constitution of the mantle, Rev. Geophys., 2, 35-88, 1964.

Cox, J.P., and R. T. Giuli, Principles of Stellar Structure, Vol. 1; Physical Principles, Gordon \& Breach, New York, 1968.
Iriyama, J., Thermal history of the earth with consideration of the formation of its core, Jour. Phys. Earth, 18, 295-311, 1970.

Knopoff, L., The convection currents, Rev. Geophys., 2, 89-122, 1964.

Lyustikh, E. N., Problem of convection in the earth's mantle, in The Earth's Crust and Uprer Mantle Geophys. Monograph 13, pp. 689-692, American Geophysical Union, Washington, D. C., 1969.

Silveston, P. L., Wärmedurchgang in waagerechten Flüssigkeitsschichten, Forch. Ing. Wes. 24, 2932, 59-69, 1958.

Tozer, D. C., Towards a theory of thermal convection in the mantle, in The Earth's Mantle, pp. 325-353, Academic Press, London, 1967.

Verhoogen, J., The adiabatic gradient in the mantle, Trans. Am. Geophys. Un., $32,41-43,1951$.

(Recieved April 23, 1971) 